\title{
A SPECTRAL METHOD FOR THE VORTICITY EQUATION ON THE SURFACE
}

\author{
GUO BEN-YU
}

\begin{abstract}
A spectral scheme is proposed for the vorticity equation defined on the spherical surface. Generalized stability and convergence are proved. The approximation results in this paper are also useful for other nonlinear problems.
\end{abstract}

\section{INTRODUCTION}

Since the spectral method has convergence rate of "infinite" order, it has become one of the most powerful tools for the numerical solution of nonlinear partial differential equations arising in fluid dynamics, e.g., see $[2,4,5,7,8,12$, $13,16,17]$. Many authors provide various spectral schemes and analyze the errors. Usually, only nonlinear problems in Descartes coordinates are considered. But in meteorological science and some other fields (see [9-11, 19]) one also has to deal with problems defined on the spherical surface. As pointed out in [2], so far, no rigorous approximation theory is available for spectral methods in spherical polar coordinates. Thus, it is desirable to develop the spectral method for spherical surfaces theoretically. In this paper, we take the vorticity equation as an example to show how to deal with such problems. In $\S 2$, we construct the scheme by using spherical harmonic functions. In $\S 3$, we list a series of lemmas which play a fundamental role in the theoretical analysis. Finally, we prove generalized stability and convergence of the scheme.

\section{THE SPECTRAL SCHEME}

Let $S$ be the unit spherical surface,

$$
S=\left\{(\lambda, \theta): 0 \leq \lambda<2 \pi,-\frac{\pi}{2} \leq \theta<\frac{\pi}{2}\right\}
$$

where $\lambda$ and $\theta$ are the longitude and latitude. Let $\xi(\lambda, \theta, t), \psi(\lambda, \theta, t)$ and $\nu>0$ be the vorticity, the stream function and the kinetic viscosity coefficient, respectively. The gradient, the Jacobi operator and the Laplace operator are as

Received by the editor October 15, 1992 and, in revised form, June 1, 1993.

1991 Mathematics Subject Classification. Primary 65N30, 76D99.

Key words and phrases. Vorticity equation, spherical surface, spectral method, approximation theory.

(C) 1995 American Mathematical Society 
follows:

$$
\begin{gathered}
\nabla \xi=\left(\frac{1}{\cos \theta} \frac{\partial \xi}{\partial \lambda}, \frac{\partial \xi}{\partial \theta}\right)^{*}, \\
J(\xi, \psi)=\frac{1}{\cos \theta}\left(\frac{\partial \xi}{\partial \lambda} \frac{\partial \psi}{\partial \theta}-\frac{\partial \xi}{\partial \theta} \frac{\partial \psi}{\partial \lambda}\right), \\
\Delta \xi=\frac{1}{\cos \theta} \frac{\partial}{\partial \theta}\left(\cos \theta \frac{\partial \xi}{\partial \theta}\right)+\frac{1}{\cos ^{2} \theta} \frac{\partial^{2} \xi}{\partial \lambda^{2}} .
\end{gathered}
$$

The vorticity equation is of the form

$$
\begin{cases}\frac{\partial \xi}{\partial t}+J(\xi, \psi)-\nu \nabla^{2} \xi=f_{1}, & (\lambda, \theta) \in S, t \in(0, T], \\ -\Delta \psi=\xi+f_{2}, & (\lambda, \theta) \in S, t \in[0, T], \\ \xi(\lambda, \theta, 0)=\xi_{0}(\lambda, \theta), & (\lambda, \theta) \in S,\end{cases}
$$

where $f_{1}, f_{2}$ and $\xi_{0}$ are given functions. It is natural to assume that all functions have period $2 \pi$ for the variable $\lambda$, and are regular at $\theta= \pm \frac{\pi}{2}$. For fixing $\psi$, we require in addition that

$$
\mu(\psi(t)) \equiv \iint_{S} \psi(\lambda, \theta, t) d S \equiv 0
$$

We shall consider the weak representation of $(2.1)$. Let $D(S)$ be the set of all infinitely differentiable functions which are regular at $\theta= \pm \frac{\pi}{2}$ and have the period $2 \pi$ in the variable $\lambda$. The duality of $D(S)$ is denoted by $D^{\prime}(S)$. We define generalized functions $u \in D^{\prime}(S)$ and their derivatives in the usual way as in [15]. Furthermore, we can define the generalized gradient, the generalized Jacobi operator and the generalized Laplace operator. For instance, if

$$
\iint_{S} u \Delta v d S=\iint_{S} v \bar{u} d S, \quad \forall v \in D(S),
$$

then the mapping $\bar{\Delta}$ such that $\bar{u}=\bar{\Delta} u$ is called the generalized Laplace operator. For simplicity, we denote $\bar{\Delta}$ by $\Delta$, etc.

Now, let

$$
L^{2}(S)=\left\{u \in D^{\prime}(S):\|u\|<\infty\right\}
$$

with the inner product and the norm being as follows:

$$
(u, v)=\iint_{S} u v d S, \quad\|u\|=(u, u)^{1 / 2} .
$$

Furthermore, let

$$
H^{1}(S)=\left\{u: u, \frac{1}{\cos \theta} \frac{\partial u}{\partial \lambda}, \frac{\partial u}{\partial \theta} \in L^{2}(S)\right\}
$$

with the following seminorm and norm:

$$
|u|_{1}=\left(\left\|\frac{1}{\cos \theta} \frac{\partial u}{\partial \lambda}\right\|^{2}+\left\|\frac{\partial u}{\partial \theta}\right\|^{2}\right)^{1 / 2}, \quad\|u\|_{1}=\left(\|u\|^{2}+|u|_{1}^{2}\right)^{1 / 2}
$$

For positive integer $r$, we can define the space $H^{r}(S)$ with the norm $\|\cdot\|_{r}$ similarly. In particular, the norm of $H^{2}(S)$ is equivalent to (see [15])

$$
\left(\|u\|^{2}+\|\Delta u\|^{2}\right)^{1 / 2} \text {. }
$$


For real $r \geq 0$, the space $H^{r}(S)$ is defined by complex interpolation between the space $H^{[r]}(S)$ and $H^{[r]+1}(S),[r]$ being the integral part of $r$. Clearly, $H^{0}(S)=L^{2}(S)$ and $\|u\|_{0}=\|u\|$. Besides, let $\|u\|_{r, \infty}=\|u\|_{C^{r}(S)}$ and $\|u\|_{\infty}=$ $\|u\|_{0, \infty}$.

It can be verified that

$$
-(\Delta u, v)=(\nabla u, \nabla v) .
$$

Thus, the weak version of $(2.1)$ is to find $(\xi, \psi) \in H^{1}(S) \times H^{1}(S)$ such that for all $v \in H^{1}(S)$,

$$
\begin{cases}\left(\frac{\partial}{\partial t} \xi(t), v\right)+(J(\xi(t), \psi(t)), v)+\nu(\nabla \xi(t), \nabla v)=\left(f_{2}(t), v\right), & t \in(0, T], \\ (\nabla \psi(t), \nabla v)=\left(\xi(t)+f_{2}(t), v\right), & t \in[0, T], \\ \xi(0)=\xi_{0} & \end{cases}
$$

The existence and uniqueness of the solution of (2.3) was discussed in [19]. Indeed, we can follow a technique similar to the proof of Theorem 6.10 in [14] to show that if $f_{1} \in L^{\infty}\left(0, T ; L^{\infty}(S)\right), f_{2} \in L^{2}\left(0, T ; L^{2}(S)\right)$ and $\xi_{0} \in L^{\infty}(S)$, then (2.3) possesses a unique solution $\xi \in L^{2}\left(0, T ; H^{1}(S)\right)$ with $\partial \xi / \partial t \in$ $L^{2}\left(0, T ; H^{-1}(S)\right), H^{-1}(S)$ being the duality of $H^{1}(S)$.

We now turn to constructing the spectral scheme for (2.3). First, let $L_{n}(z)$ be the Legendre polynomial of degree $n$,

$$
L_{n}(z)=\frac{1}{2^{n} n !} \frac{d^{n}}{d z^{n}}\left(z^{2}-1\right)^{n} .
$$

The normalized associated Legendre function is defined as

$$
\begin{aligned}
& L_{m, n}(z)=\sqrt{\frac{(2 n+1)(n-m) !}{2(n+m) !}}\left(1-z^{2}\right)^{m / 2} \frac{d^{m}}{d z^{m}} L_{n}(z), \quad m \geq 0, n \geq|m|, \\
& L_{m, n}(z)=L_{-m, n}(z), \quad m<0, n \geq|m| .
\end{aligned}
$$

Furthermore, the spherical harmonic $Y_{m, n}(\lambda, \theta)$ is

$$
Y_{m, n}(\lambda, \theta)=\frac{1}{\sqrt{2 \pi}} e^{i m \lambda} L_{m, n}(\sin \theta), \quad n \geq|m| .
$$

It can be verified that (see [3])

$$
-\Delta Y_{m, n}(\lambda, \theta)=n(n+1) Y_{m, n}(\lambda, \theta)
$$

and

$$
\int_{0}^{2 \pi} \int_{-\pi / 2}^{\pi / 2} Y_{m, n}(\lambda, \theta) Y_{m^{\prime}, n^{\prime}}^{*}(\lambda, \theta) \cos \theta d \theta d \lambda= \begin{cases}1 & \text { if } m=m^{\prime}, n=n^{\prime} \\ 0 & \text { otherwise. }\end{cases}
$$

We set

$$
\hat{u}_{m, n}=\int_{0}^{2 \pi} \int_{-\pi / 2}^{\pi / 2} u(\lambda, \theta) Y_{m, n}^{*}(\lambda, \theta) \cos \theta d \theta d \lambda
$$

Let

$$
\widetilde{V}_{M}=\operatorname{span}\left\{Y_{m, n}:|m| \leq M,|m| \leq n \leq N(m)\right\},
$$

where $N(m)$ determines the construction of spectral approximation. Usually we take $N(m)=M$ or $N(m)=M+|m|$. For simplicity, suppose $N(m)=M$. Let $V_{M}$ be the subset of $\widetilde{V}_{M}$ containing all real-valued functions. 
Let $P_{M}$ be the orthogonal projection from $L^{2}(S)$ onto $V_{M}$ such that for any $u \in L^{2}(S)$

or equivalently,

$$
\left(P_{M} u-u, v\right)=0, \quad \forall v \in V_{M},
$$

$$
P_{M} u=\sum_{|m| \leq M} \sum_{n \geq|m|} \hat{u}_{m, n} Y_{m, n}(\lambda, \theta) .
$$

Let $\tau$ be the mesh size in the variable $t$, and

Define

$$
\dot{R}_{\tau}=\left\{t=k \tau: 1 \leq k \leq\left[\frac{T}{\tau}\right]\right\}, \quad R_{\tau}=\dot{R}_{\tau} \cup\{0\} .
$$

$$
\delta_{\tau} u(\lambda, \theta, t)=\frac{1}{\tau}(u(\lambda, \theta, t+\tau)-u(\lambda, \theta, t)) .
$$

Let $(\eta, \varphi) \in V_{M} \times V_{M}$ be the approximation to $(\xi, \psi)$ and $b$ and $\sigma$ parameters with $0 \leq b, \sigma \leq 1$. The spectral scheme for (2.3) is as follows:

$$
\begin{cases}\left(\delta_{\tau} \eta(t), v\right)+\left(J\left(\eta(t)+b \tau \delta_{\tau} u(t), \varphi(t)\right), v\right) & \\ -\nu\left(\Delta\left(\eta(t)+\sigma \tau \delta_{\tau} \eta(t)\right), v\right)=\left(f_{1}(t), v\right), & \forall v \in V_{M}, t \in \dot{R}_{\tau}, \\ -(\Delta \varphi(t), v)=\left(\eta(t)+f_{2}(t), v\right), & \forall v \in V_{M}, t \in R_{\tau}, \\ \mu(\varphi(t))=0, & \forall t \in R_{\tau}, \\ \eta(0)=P_{M} \xi_{0} . & \end{cases}
$$

Clearly, if $b=\sigma=0$, then (2.5) is an explicit scheme. If $b=0$ and $\sigma \neq 0$, we still can obtain the value of $\eta(t)$ explicitly by the orthogonality of spherical harmonic functions. Indeed, this is one of the advantages of spectral methods. Otherwise, the scheme is implicit, and so an iteration is needed for evaluating $\eta(t)$ at each $t \in \dot{R}_{\tau}$.

We now consider the existence and uniqueness of the solution of (2.5) with $b \neq 0$. We have

$$
\begin{gathered}
(\eta(t), v)+b \tau(J(\eta(t), \varphi(t-\tau)), v)-\nu \sigma \tau(\Delta \eta(t), v) \\
=(F(t), v), \quad \forall v \in V_{m}, t \in \dot{R}_{\tau},
\end{gathered}
$$

where

$$
\begin{gathered}
F(t)=\eta(t-\tau)-\tau(1-b) J(\eta(t-\tau), \varphi(t-\tau)) \\
+\nu \tau(1-\sigma) \Delta \eta(t-\tau)+\tau f_{1}(t-\tau) .
\end{gathered}
$$

Clearly, this is a linear algebraic system for the unknown coefficients of the spherical harmonic expansion of $\eta(t)$. Thus, we only have to show that the equation

$$
(Z, v)+b \tau(J(Z, \varphi), v)-\nu \sigma \tau(\Delta Z, v)=0
$$

has only the trivial solution. By taking $v=z$, we obtain from Lemma 6 (see $\S 3$ of this paper) that

$$
\|Z\|^{2}+\nu \sigma \tau[Z]_{1}^{2}=0
$$

and thus $Z \equiv 0$. Therefore, $\eta(t)$ is determined uniquely at each time $t \in \dot{R}_{\tau}$.

\section{Some Lemmas}

For analyzing the errors, we need some basic estimates. In this section, we prove several lemmas. Throughout this paper, we denote by $c$ a positive constant independent of $M, \tau$ and any function, which may be different in different occurrences. The notation " $\subseteq$ " means the embedding of spaces. 
Lemma 1. We have $H^{\beta}(S) \subseteq H^{r}(S)$ for $0 \leq r \leq \beta$ and $H^{1+\beta}(S) \subset C(S)$ for $\beta>0$.

Proof. The first assertion follows directly from the definition. We now prove the second one. Let $B$ be the unit ball in the three-dimensional Euclidean space, and $w$ a function defined on $B$. We denote by $\gamma(w)$ the restriction of $w$ on $S$. We can take $H^{1+\beta}(S)$ to be the trace space of $H^{3 / 2+\beta}(B)$ equipped with the norm

$$
\|u\|_{H^{1+\beta}(S)}=\inf _{\substack{w \in H^{3 / 2+\beta}(B) \\ \gamma(w)=u}}\|w\|_{H^{3 / 2+\beta}(B)} .
$$

By embedding theory, $H^{3 / 2+\beta}(B) \subseteq C(B)$, and so for any $w \in H^{3 / 2+\beta}(B)$,

$$
\|w\|_{C(B)} \leq c\|w\|_{H^{3 / 2+\beta}(B)} .
$$

On the other hand, for any $u \in H^{1+\beta}(S)$, there exists $\bar{w} \in H^{3 / 2+\beta}(B)$ such that $\gamma(\bar{w})=u$ and

Therefore,

$$
\|u\|_{H^{1+\beta}(S)} \geq \frac{1}{2}\|\bar{w}\|_{H^{3 / 2+\beta}(B)} .
$$

$$
\begin{aligned}
\|u\|_{C(S)}=\sup _{x \in S}|u(x)| & =\sup _{x \in S}|\bar{w}(x)| \\
& \leq \sup _{x \in B}|\bar{w}(x)| \leq c\|\bar{w}\|_{H^{3 / 2+\beta}(B)} \leq 2 c\|u\|_{H^{1+\beta}(S)},
\end{aligned}
$$

which implies the second assertion.

Lemma 2. There exists a positive constant $c$ such that $\|u\| \leq c|u|_{1}$ for all $u \in$ $H^{1}(S)$ with $\mu(u)=0$.

Proof. By the Poincare inequality, we have

$$
\|u\|^{2} \leq c^{2}\left(\mu(u)+|u|_{1}^{2}\right),
$$

and so the claim follows.

Lemma 3. If $u \in L^{2}(S), v \in H^{1+\beta}(S)$ and $\beta>0$, then

Proof. By Lemma 1,

$$
\|u v\| \leq c\|u\|\|v\|_{1+\beta} \text {. }
$$

$$
\|u v\| \leq c\|u\|^{2}\|v\|_{\infty}^{2} \leq c\|u\|^{2}\|v\|_{1+\beta}^{2} .
$$

Lemma 4. If $u \in V_{M}$ and $0 \leq r \leq \beta$, then

Proof. Let

$$
\|u\|_{\beta} \leq c M^{\beta-r}\|u\|_{r} .
$$

$$
u=\sum_{m=-M}^{M} \sum_{n=|m|}^{M} \hat{u}_{m, n} Y_{m, n}(\lambda, \theta) .
$$

By (2.4), $Y_{m, n}(\lambda, \theta)$ is the eigenfunction of the operator $-\Delta$ on $S$, corresponding to the eigenvalue $n(n+1)$. Thus, for the space $H^{r}(s)$, the norm $\|v\|_{r}$ is equivalent to (see [15])

$$
\left(\sum_{m=-\infty}^{\infty} \sum_{n \geq|m|} n^{r}(n+1)^{r}\left|\hat{v}_{m, n}\right|^{2}\right)^{1 / 2} .
$$


Then

$$
\begin{aligned}
\|u\|_{\beta}^{2} & \leq c \sum_{m=-M}^{M} \sum_{n=|m|}^{M} n^{\beta}(n+1)^{\beta}\left|\hat{u}_{m, n}\right|^{2} \\
& \leq c M^{2 \beta-2 r} \sum_{m=-M}^{M} \sum_{n=|m|}^{M} n^{r}(n+1)^{r}\left|\hat{u}_{m, n}\right|^{2} \\
& \leq c M^{2 \beta-2 r}\|u\|_{r}^{2} .
\end{aligned}
$$

Lemma 5. If $u \in H^{\beta}(S)$ and $r \leq \beta$, then

Proof. By (3.1),

$$
\begin{aligned}
& \left\|u-P_{M} u\right\|_{r} \leq c M^{r-\beta}\|u\|_{\beta}, \\
& \left\|P_{M} u\right\|_{r} \leq c\|u\|_{r} .
\end{aligned}
$$

$$
\begin{aligned}
\left\|u-P_{M} u\right\|_{r}^{2} & \leq c \sum_{m=-M}^{M} \sum_{n=M+1}^{\infty} n^{r}(n+1)^{r}\left|\hat{u}_{m, n}\right|^{2}+c \sum_{|m|>M} \sum_{n=|m|}^{\infty} n^{r}(n+1)^{r}\left|\hat{u}_{m, n}\right|^{2} \\
& \leq c \sum_{m=-M}^{M} \sum_{n=M+1}^{\infty} n^{r}(n+1)^{r}\left|\hat{u}_{m, n}\right|^{2}+c \sum_{|m|>M} \sum_{n=M+1}^{\infty} n^{r}(n+1)^{r}\left|\hat{u}_{m, n}\right|^{2} \\
& \leq c M^{2 r-2 \beta} \sum_{m=-\infty}^{\infty} \sum_{n=M+1}^{\infty} n^{\beta}(n+1)^{\beta}\left|\hat{u}_{m, n}\right|^{2} \\
& \leq c M^{2 r-2 \beta}\|u\|_{r}^{2} .
\end{aligned}
$$

The second inequality follows from the first with $\beta=r$ by using the triangle inequality.

Lemma 3 and Lemma 4 are the approximation and inverse inequalities for spherical harmonics. In [1], Bramble and Pasciak gave similar results with different proofs.

Lemma 6. If $u, v \in H^{1+\beta}(S), w \in H^{1}(S)$ and $\beta>0$, then

$$
(u, J(v, w))+(v, J(u, w))=0 .
$$

Proof. We have

$$
\begin{aligned}
(u, J(v, w))= & \int_{0}^{2 \pi} \int_{-\pi / 2}^{\pi / 2} u\left(\frac{\partial v}{\partial \lambda} \frac{\partial w}{\partial \theta}-\frac{\partial v}{\partial \theta} \frac{\partial w}{\partial \lambda}\right) d \theta d \lambda \\
= & -\int_{0}^{2 \pi} \int_{-\pi / 2}^{\pi / 2} v\left(\frac{\partial u}{\partial \lambda} \frac{\partial w}{\partial \theta}-\frac{\partial u}{\partial \theta} \frac{\partial w}{\partial \lambda}\right) d \theta d \lambda \\
& -\int_{0}^{2 \pi} u\left(\lambda, \frac{\pi}{2}\right) v\left(\lambda, \frac{\pi}{2}\right) \frac{\partial w}{\partial \lambda}\left(\lambda, \frac{\pi}{2}\right) d \lambda \\
& +\int_{0}^{2 \pi} u\left(\lambda,-\frac{\pi}{2}\right) v\left(\lambda,-\frac{\pi}{2}\right) \frac{\partial w}{\partial \lambda}\left(\lambda,-\frac{\pi}{2}\right) d \lambda
\end{aligned}
$$

We know from the regularity of $w$ that $w$ approaches the limits independently of $\lambda$, as $\theta \rightarrow \pm \pi / 2$ (see p. 314 of [3]). This means that $\partial w / \partial \lambda=0$ at $\theta= \pm \pi / 2$, and so the conclusion follows. 
Lemma 7 (see [6]). For any $u \in C\left(0, T ; L^{2}(S)\right)$

$$
2\left(\delta_{\tau} u(t), u(t)\right)=\delta_{\tau}\|u(t)\|^{2}-\tau\left\|\delta_{\tau} u(t)\right\|^{2} .
$$

Lemma 8 (see [6]). Assume that

(i) $E(t)$ is a nonnegative function defined on $R_{\tau}$,

(ii) $\rho, b_{1}, b_{2}, d_{1}$ and $d_{2}$ are nonnegative constants,

(iii) for $t \in \dot{R}_{\tau}$

$$
E(t) \leq \rho+\tau \sum_{t^{\prime}=0}^{t-\tau}\left(d_{1} E\left(t^{\prime}\right)+d_{2} M^{b_{1}} E^{b_{2}+1}\left(t^{\prime}\right)\right),
$$

(iv) $E(0) \leq \rho$ and $\rho e^{\left(d_{1}+d_{2}\right) t_{1}} \leq M^{-b_{1} / b_{2}}$.

Then for all $t \in R_{\tau}$ and $t \leq t_{1}$

$$
E(t) \leq \rho e^{\left(d_{1}+d_{2}\right) t} .
$$

If in addition $d_{2}=0$, then for all $\rho$ and $t \leq T$

$$
E(t) \leq \rho e^{d_{1} t} .
$$

\section{Generalized Stability OF THE SCHEME}

As is known, nonlinear schemes are usually not stable in the sense of Lax, but might be so in the sense of generalized stability $[6,18]$. We now analyze the generalized stability of scheme (2.5). Suppose that $\eta(0), f_{1}$ and $f_{2}$ have errors $\tilde{\eta}_{0}, \tilde{f}_{1}$ and $\tilde{f}_{2}$, respectively. They induce errors of $\eta$ and $\varphi$, denoted by $\tilde{\eta}$ and $\tilde{\varphi}$. Then

$$
\begin{cases}\left(\delta_{\tau} \tilde{\eta}(t), v\right)+\left(J \left(\tilde{\eta}(t)+b \tau \delta_{\tau} \tilde{\eta}(t),\right.\right. & \varphi(t)+\tilde{\varphi}(t)), v) \\ +\left(J\left(\eta(t)+b \tau \delta_{\tau} \eta(t), \tilde{\varphi}(t)\right), v\right)-\nu\left(\Delta\left(\tilde{\eta}(t)+\sigma \tau \delta_{\tau} \tilde{\eta}_{t}(t)\right), v\right) & \\ =\left(\tilde{f}_{1}(t), v\right), & \forall v \in V_{M}, t \in \dot{R}_{\tau}, \\ -(\Delta \tilde{\varphi}(t), v)=\left(\tilde{\eta}(t)+\tilde{f}_{2}(t), v\right), & \forall v \in V_{M}, t \in R_{\tau}, \\ \mu(\tilde{\varphi}(t))=0, & t \in R_{\tau}, \\ \tilde{\eta}(0)=\tilde{\eta}_{0} . & \end{cases}
$$

By taking $v=2 \tilde{\eta}$ in the first formula of (4.1), we have from Lemmas 6 and 7 that

$$
\begin{gathered}
\delta_{\tau}\|\tilde{\eta}(t)\|^{2}-\tau\left\|\delta_{\tau} \tilde{\eta}(t)\right\|^{2}+2 \nu|\tilde{\eta}(t)|_{1}^{2}+\nu \sigma \tau \delta_{\tau}|\tilde{\eta}(t)|_{1}^{2}-\nu \sigma \tau^{2}\left|\delta_{\tau} \tilde{\eta}(t)\right|_{1}^{2}+F_{1}(t) \\
-2 b \tau\left(J(\tilde{\eta}(t), \varphi(t)+\tilde{\varphi}(t)), \delta_{\tau} \tilde{\eta}(t)\right)=2\left(\tilde{f}_{1}(t), \tilde{\eta}(t)\right)
\end{gathered}
$$

where

$$
F_{1}(t)=2\left(J\left(\eta(t)+b \tau \delta_{\tau} \eta(t), \tilde{\varphi}(t)\right), \tilde{\eta}(t)\right) .
$$

Next, let $d$ be an undetermined constant. By taking $v=d \tau \delta_{\tau} \tilde{\eta}$ in the same formula, we get

$$
\begin{gathered}
d \tau\left\|\delta_{\tau} \tilde{\eta}(t)\right\|^{2}-\frac{1}{2} \nu d \tau \delta_{\tau}|\tilde{\eta}(t)|_{1}^{2}+\nu d \tau^{2}\left(\sigma-\frac{1}{2}\right)\left|\delta_{\tau} \tilde{\eta}(t)\right|_{1}^{2}+F_{2}(t) \\
+d \tau\left(J(\tilde{\eta}(t), \varphi(t)+\tilde{\varphi}(t)), \delta_{\tau} \tilde{\eta}(t)\right)=d \tau\left(\tilde{f}_{1}(t), \delta_{\tau} \tilde{\eta}(t)\right)
\end{gathered}
$$

where

$$
F_{2}(t)=d \tau\left(J\left(\eta(t)+b \tau \delta_{\tau} \eta(t), \tilde{\varphi}(t)\right), \delta_{\tau} \tilde{\eta}(t)\right)
$$


Putting (4.2) and (4.3) together, we obtain

$$
\begin{aligned}
& \delta_{\tau}\|\tilde{\eta}(t)\|^{2}+\tau(d-1)\left\|\delta_{\tau} \tilde{\eta}(t)\right\|^{2}+2 \nu|\tilde{\eta}(t)|_{1}^{2}+\nu \tau\left(\sigma+\frac{d}{2}\right) \delta_{\tau}|\tilde{\eta}(t)|_{1}^{2} \\
& +\nu \tau^{2}\left(d \sigma-\sigma-\frac{d}{2}\right)\left|\delta_{\tau} \tilde{\eta}(t)\right|_{1}^{2}+\sum_{j=1}^{4} F_{j}(t)=\left(\tilde{f}_{1}(t), 2 \tilde{\eta}(t)+d \tau \delta_{\tau} \tilde{\eta}(t)\right),
\end{aligned}
$$

where

$$
\begin{aligned}
& F_{3}(t)=\tau(d-2 b)\left(J(\tilde{\eta}(t), \varphi(t)), \delta_{\tau} \tilde{\eta}(t)\right), \\
& F_{3}(t)=\tau(d-2 b)\left(J(\tilde{\eta}(t), \tilde{\varphi}(t)), \delta_{\tau} \tilde{\eta}(t)\right) .
\end{aligned}
$$

Furthermore, we put $v=\tilde{\varphi}$ in the second formula of (4.1) and obtain

$$
|\tilde{\varphi}(t)|_{1}^{2} \leq \frac{1}{2 c}\|\tilde{\varphi}(t)\|^{2}+c\left(\|\tilde{\eta}(t)\|^{2}+\left\|\tilde{f}_{2}(t)\right\|^{2}\right) .
$$

Thus, Lemma 2 leads to

$$
|\tilde{\varphi}(t)|_{1}^{2} \leq c\left(\|\tilde{\eta}(t)\|^{2}+\left\|\tilde{f}_{2}(t)\right\|^{2}\right) .
$$

Moreover, by Lemma 2 and (4.5),

$$
\|\tilde{\varphi}(t)\|_{2}^{2} \leq c\left(\|\tilde{\varphi}(t)\|^{2}+\|\Delta \tilde{\varphi}(t)\|^{2}\right) \leq c\left(\|\tilde{\eta}(t)\|^{2}+\left\|\tilde{f}_{2}(t)\right\|^{2}\right) .
$$

We now estimate the $\left|F_{j}(t)\right|$. Let $\varepsilon>0$ and

$$
\|u \mid\|_{r}=\max _{0 \leq t \leq T}\|u(t)\|_{r}, \quad\|\| u\left\|_{r, \infty}=\max _{0 \leq t \leq T}\right\| u(t) \|_{r, \infty} \text {, etc. }
$$

By Lemma 1, Lemma 6 and (4.5), we know that for any $\beta>0$,

$$
\begin{aligned}
\left|F_{1}(t)\right| & =\left|2\left(J(\tilde{\eta}(t), \tilde{\varphi}(t)), \eta(t)+b \tau \delta_{\tau} \eta(t)\right)\right| \\
& \leq \varepsilon \nu|\tilde{\eta}(t)|_{1}^{2}+\left.\frac{c}{\varepsilon \nu}|\| \eta|\right|_{\infty} ^{2}|\tilde{\varphi}(t)|_{1}^{2} \\
& \leq \varepsilon \nu|\tilde{\eta}(t)|_{1}^{2}+\frac{c}{\varepsilon \nu}\left\||\eta \||_{1+\beta}^{2}\left(\|\tilde{\eta}(t)\|^{2}+\left\|\tilde{f}_{2}(t)\right\|^{2}\right) .\right.
\end{aligned}
$$

Similarly,

$$
\left|F_{2}(t)\right| \leq \varepsilon \nu \tau^{2}\left|\delta_{\tau} \tilde{\eta}(t)\right|_{1}^{2}+\frac{c d^{2}}{\varepsilon \nu}\|\| \eta \|\left.\right|_{1+\beta} ^{2}\left(\|\tilde{\eta}(t)\|^{2}+\left\|\tilde{f}_{2}(t)\right\|^{2}\right) .
$$

Furthermore, Lemma 3 leads to

$$
\left|F_{3}(t)\right| \leq \varepsilon \tau\left\|\delta_{\tau} \tilde{\eta}(t)\right\|^{2}+\frac{c \tau(d-2 b)^{2}}{\varepsilon}|\|\varphi\||_{2+\beta}^{2}|\tilde{\eta}(t)|_{1}^{2} .
$$

Since

$$
\|\varphi(t)\|_{2}^{2} \leq c\left(\|\varphi(t)\|^{2}+\|\Delta \varphi(t)\|^{2}\right) \leq c\left(\|\eta(t)\|^{2}+\left\|f_{2}(t)\right\|^{2}\right),
$$

we have from Lemma 4 that

$$
\left|F_{3}(t)\right| \leq \varepsilon \tau\left\|\delta_{\tau} \tilde{\eta}(t)\right\|^{2}+\frac{c \tau M^{\beta}(d-2 b)^{2}}{\varepsilon}\left(\|\| \eta\left\|\left.\right|^{2}+||\left|f_{2}\right|\right\|^{2}\right)|\tilde{\eta}(t)|_{1}^{2} .
$$


Also, by Lemma 3, Lemma 4 and the second formula of (4.1), we obtain

$$
\begin{aligned}
\left|F_{4}(t)\right| & \leq \varepsilon \tau\left\|\delta_{\tau} \tilde{\eta}(t)\right\|^{2}+\frac{c \tau(d-2 b)^{2}}{\varepsilon}\|\tilde{\varphi}(t)\|_{2+\beta}^{2}|\tilde{\eta}(t)|_{1}^{2} \\
& \leq \varepsilon \tau\left\|\delta_{\tau} \tilde{\eta}(t)\right\|^{2}+\frac{c \tau(d-2 b)^{2}}{\varepsilon}\left(\|\tilde{\eta}(t)\|_{\beta}^{2}+\left\|\tilde{f}_{2}(t)\right\|_{\beta}^{2}\right)|\tilde{\eta}(t)|_{1}^{2} \\
& \leq \varepsilon \tau\left\|\delta_{\tau} \tilde{\eta}(t)\right\|^{2}+\frac{c \tau M^{\beta}(d-2 b)^{2}}{\varepsilon}\left(\|\tilde{\eta}(t)\|^{2}+\left\|\tilde{f}_{2}(t)\right\|^{2}\right)|\tilde{\eta}(t)|_{1}^{2} .
\end{aligned}
$$

Finally,

$$
\left|\left(\tilde{f}_{1}(t), \tilde{\eta}(t)+d \tau \delta_{\tau} \tilde{\eta}(t)\right)\right| \leq \varepsilon \tau\left\|\delta_{\tau} \tilde{\eta}(t)\right\|^{2}+c\|\tilde{\eta}(t)\|^{2}+c\left(1+\frac{\tau d^{2}}{\varepsilon}\right)\left\|\tilde{f}_{1}(t)\right\|^{2} .
$$

By substituting the above estimates into (4.4), we obtain

$$
\begin{gathered}
\delta_{\tau}\|\tilde{\eta}(t)\|^{2}+\tau(d-1-3 \varepsilon)\left\|\delta_{\tau} \tilde{\eta}(t)\right\|^{2}+\nu|\tilde{\eta}(t)|_{1}^{2}+\nu \tau\left(\sigma+\frac{d}{2}\right) \delta_{\tau}|\tilde{\eta}(t)|_{1}^{2} \\
+A_{0} \tau\left|\delta_{\tau} \tilde{\eta}(t)\right|_{1}^{2} \leq A_{1}\|\tilde{\eta}(t)\|^{2}+A_{2}(t)|\tilde{\eta}(t)|_{1}^{2}+A_{3}(t)
\end{gathered}
$$

where

$$
\begin{aligned}
& A_{0}=\nu \tau\left(d \sigma-\sigma-\frac{d}{2}-\varepsilon\right), \\
& A_{1}=c\left(1+\frac{1+d^{2}}{\varepsilon \nu}\|\| \eta \|_{1+\beta}^{2}\right), \\
& A_{2}(t)=-\nu+\varepsilon+\frac{c \tau M^{\beta}}{\varepsilon}(d-2 b)^{2}\left(\|\| \eta\left\|^{2}+\right\|\left\|f_{2}\right\|\left\|^{2}+\right\| \tilde{\eta}(t)\left\|^{2}+\right\| \tilde{f}_{2}(t) \|^{2}\right), \\
& A_{3}(t)=c\left(1+\frac{\tau d^{2}}{\varepsilon}\right)\left\|\tilde{f}_{1}(t)\right\|^{2}+\frac{c}{\varepsilon \nu}\left(1+d^{2}\right)\left\|\tilde{f}_{2}(t)\right\|^{2} .
\end{aligned}
$$

Let $p_{0}>0$ and $\varepsilon$ be suitably small. We choose the value of $d$ in three different cases as follows:

(i) $\sigma>1 / 2$. We take

$$
d \geq d_{1}=\max \left(1+3 \varepsilon+p_{0}, \frac{2 \sigma+2 p_{0}+2 \varepsilon}{2 \sigma-1}\right) .
$$

Then

$$
\tau(d-1-3 \varepsilon)\left\|\tilde{\eta}_{t}(t)\right\|^{2}+A_{0} \tau\left|\tilde{\eta}_{t}(t)\right|_{1}^{2} \geq p_{0} \tau\left\|\tilde{\eta}_{t}(t)\right\|^{2} .
$$

(ii) $\sigma=\frac{1}{2}$. According to Lemma 4, there exists a positive constant $q$ such that $|u|_{1}^{2} \leq q M^{2}\|u\|^{2}$ for any $u \in V_{M}$. We take

$$
d \geq d_{2}=1+3 \varepsilon+p_{0}+\nu q \tau M^{2}\left(\frac{1}{2}+\varepsilon\right),
$$

and so (4.7) holds again.

(iii) $\sigma<\frac{1}{2}$ and $\tau M^{2}<\frac{2}{q \nu(1-2 \sigma)}$. Then we take

$$
d \geq d_{3}=\frac{1+3 \varepsilon+p_{0}+q \nu \tau M^{2}(\sigma+\varepsilon)}{1+q \nu \tau M^{2}\left(\sigma-\frac{1}{2}\right)} .
$$

It can be verified that (4.7) is still fulfilled. 
Now, let

$$
\begin{aligned}
& E(t)=\|\tilde{\eta}(t)\|^{2}+\tau \sum_{z=0}^{t-\tau}\left(\nu|\tilde{\eta}(z)|_{1}^{2}+p_{0} \tau\left\|\delta_{\tau} \tilde{\eta}(z)\right\|^{2}\right) \\
& \rho(t)=\|\tilde{\eta}(0)\|^{2}+\nu \tau\left(\sigma+\frac{d}{2}\right)|\tilde{\eta}(0)|_{1}^{2}+\tau \sum_{z=0}^{t-\tau} A_{3}(z)
\end{aligned}
$$

By summing up (4.6) for $t \in R_{\tau}$, we obtain

$$
E(t) \leq \rho(t)+\tau \sum_{z=0}^{t-\tau}\left(A_{1} E(z)+A_{2}(z) E^{2}(z)\right)
$$

Finally, by applying Lemma 8 to (4.8), we get the following result.

Theorem 1. Assume that

(i) $\tau M^{\beta}$ is suitably small, $\beta$ being an arbitrarily small positive constant,

(ii) $\sigma>\frac{1}{2}$ or $\tau M^{2}<\frac{2}{q \nu(1-2 \sigma)}$,

(iii) $\left\|\tilde{f}_{2}(t)\right\| \leq b_{1}$ and $\rho(t) \leq b_{2}$ for $t \leq t_{1}$,

where $t_{1} \leq T, b_{1}$ and $b_{2}$ are suitably small constants depending only on \|\|$\eta\left\|_{1+\beta},\right\||| f_{2} \| \mid$ and $\nu$. Then for all $t \leq t_{1}$

$$
E(t) \leq \rho(t) e^{b_{3} t}
$$

We now consider a special case, i.e.,

$$
\begin{cases}2 b \geq d_{1} & \text { for } \sigma>\frac{1}{2} \\ 2 b \geq d_{2} & \text { for } \sigma=\frac{1}{2} \\ 2 b \geq d_{3} & \text { for } \sigma<\frac{1}{2}\end{cases}
$$

Then we can take $d=2 b$ and so $A_{2}=-\nu$. Thus, the following conclusion follows.

Theorem 2. Let $\sigma>\frac{1}{2}$ or $\tau M^{2}<\frac{1}{q \nu(1-2 \sigma)}$. If in addition (4.10) is fulfilled, then (4.9) holds for all $\tilde{f}_{2}(t), \rho(t)$ and $t \leq T$.

\section{Convergence}

In this section, we deal with convergence. By (2.4),

$$
\begin{aligned}
-P_{M} \Delta u(\lambda, \theta) & =\sum_{|m| \leq M} \sum_{n \geq|m|} n(n+1) \hat{u}_{m, n} Y_{m, n}(\lambda, \theta) \\
& =-\sum_{|m|<M} \sum_{n \geq|m|} \hat{u}_{m, n} \Delta Y_{m, n}(\lambda, \theta)=-\Delta P_{M} u(\lambda, \theta) .
\end{aligned}
$$

Let $\xi^{(M)}=P_{M} \xi$ and $\psi^{(M)}=P_{M} \psi$. Then from (2.3) we obtain that for all $v \in V_{M}$ 


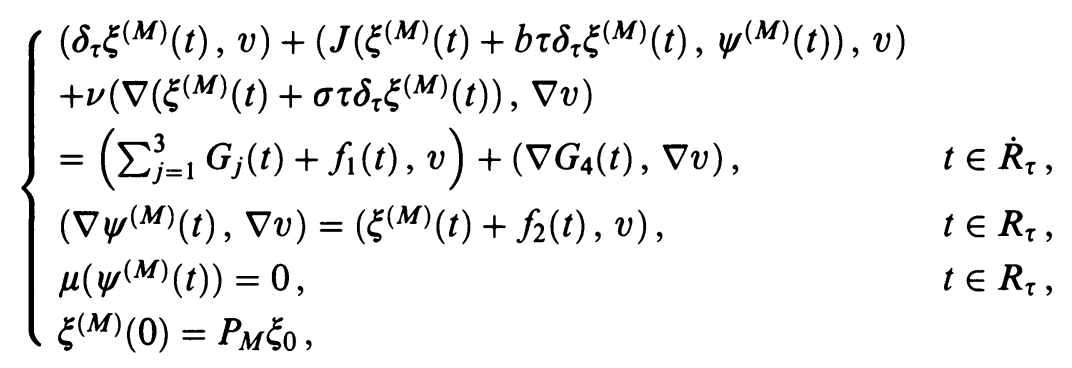

where

$$
\begin{aligned}
& G_{1}(t)=\delta_{\tau} \xi^{(M)}(t)-\frac{\partial}{\partial t} \xi^{(M)}(t), \\
& G_{2}(t)=b \tau J\left(\delta_{\tau} \xi^{(M)}(t), \psi^{(M)}(t)\right), \\
& G_{3}(t)=J\left(\xi^{(M)}(t), \psi^{(M)}(t)\right)-J(\xi(t), \psi(t)), \\
& G_{4}(t)=\sigma \tau \delta_{\tau} \xi^{(M)}(t) .
\end{aligned}
$$

Next, let $\eta=\xi^{(M)}+\tilde{\xi}$ and $\varphi=\psi^{(M)}+\tilde{\psi}$. Then we have from (2.5) and (5.1) that for all $v \in V_{M}$

$$
\begin{cases}\left(\delta_{\tau} \tilde{\xi}(t), v\right)+\left(J\left(\tilde{\xi}(t)+b \tau \delta_{\tau} \tilde{\xi}(t), \psi^{(M)}(t)+\tilde{\psi}(t)\right), v\right) & \\ +\left(J\left(\xi^{(M)}(t)+b \tau \delta_{\tau} \xi^{(M)}(t), \tilde{\psi}(t)\right), v\right)+\nu\left(\Delta\left(\tilde{\xi}(t)+\sigma \tau \delta_{\tau} \tilde{\xi}(t)\right), v\right) & \\ =-\left(\sum_{j=1}^{3} G_{j}(t), v\right)-\left(\nabla G_{4}(t), \nabla v\right), & t \in \dot{R}_{\tau}, \\ (\Delta \tilde{\psi}(t), v)=(\tilde{\xi}(t), v), & t \in R_{\tau}, \\ \mu(\tilde{\psi}(t))=0, & t \in R_{\tau}, \\ \tilde{\xi}(0)=0 . & \end{cases}
$$

Clearly, we can get the same estimate as in (4.9). But $\eta$ and $\tilde{\eta}$ are replaced by $\xi$ and $\tilde{\xi}$, respectively, and $\rho(t)$ is replaced by

$$
\tilde{\rho}(t)=\tau \sum_{z=0}^{t-\tau}\left(\left\|G_{1}(z)\right\|^{2}+\left\|G_{1}(z)\right\|^{2}+\left\|G_{3}(z)\right\|^{2}+\left|G_{4}(z)\right|_{1}^{2}\right) .
$$

\section{According to}

$$
\delta_{\tau} \xi(t)-\frac{\partial \xi}{\partial t}(t)=\frac{1}{\tau} \int_{t}^{t-\tau}(t+\tau-z) \frac{\partial^{2} \xi}{\partial z^{2}}(z) d z
$$

we have

$$
\tau \sum_{z=0}^{t-\tau}\left\|G_{1}(z)\right\|^{2} \leq c \tau^{2}\|\xi\|_{H^{2}\left(0, t ; L^{2}(S)\right)}^{2}
$$

By Lemma 3 and Lemma 5,

$$
\begin{aligned}
\left\|G_{2}(t)\right\|^{2} & \leq c \tau^{2}\left\|\left|\psi^{(M)}\right|\right\|_{2+\beta}\left\|\frac{\partial \xi^{(M)}}{\partial t}(t)\left|\left\|_{1}^{2} \leq c \tau^{2}\right\|\right| \psi\left|\left\|_{2+\beta}\right\| \frac{\partial \xi}{\partial t} \|\right|_{1}^{2}\right. \\
& \leq c \tau^{2}\left(\|\xi\|_{\beta}^{2}+\left.\left\||| f_{2}\right\|\right|_{\beta} ^{2}\right)\left|\left\|\frac{\partial \xi}{\partial t}\right\|\right|_{1}^{2} .
\end{aligned}
$$


Furthermore, for $r>0$,

$$
\begin{aligned}
& \left\|G_{3}(t)\right\|^{2} \leq c\left\|\psi^{(M)}(t)\right\|_{2+\beta}\left\|\xi^{(M)}(t)-\xi(t)\right\|_{1}^{2}+c\left\|\xi^{(M)}(t)\right\|_{1}\left\|\psi^{(M)}(t)-\psi(t)\right\|_{2+\beta}^{2} \\
& \leq c M^{-2 r}\left(\|\| \psi\left\|\left.\right|_{2+\beta} ^{2}\right\||\xi|\left\|_{1+r}^{2}+\right\||\xi|\left\|_{1}^{2}\right\||\psi| \|_{2+\beta+r}^{2}\right) \\
& \leq c M^{-2 r}\left[\left(\| | \xi | | _ { \beta } ^ { 2 } + | | | f _ { 2 } \| | _ { \beta } ^ { 2 } ) \left|\left\|\xi||_{1+r}^{2}+\left(\left.|||\xi|\right|_{\beta+r} ^{2}+\left|\left\|f_{2}\right\|\right|_{\beta+r}^{2}\right)\left|\|\xi \mid\|_{1}^{2}\right] .\right.\right.\right.\right.
\end{aligned}
$$

Finally,

$$
\left|G_{4}(t)\right|_{1}^{2} \leq c \tau^{2}\left\|\frac{\partial \xi}{\partial t}\right\| \|_{1}^{2} .
$$

Therefore, $\tilde{\rho}(t)=O\left(\tau^{2}+M^{-2 r}\right)$, and so we obtain the following results.

Theorem 3. Let $r>0$ and $\beta$ be arbitrarily small positive constants. Assume that

(i) (4.10) or condition (i) of Theorem 1 holds,

(ii) condition (ii) of Theorem 1 is satisfied,

(iii) $\xi \in C\left(0, T ; H^{1+r}(S)\right) \cap C^{1}\left(0, T ; H^{1}(S)\right) \cap H^{2}\left(0, T ; L^{2}(S)\right)$ and $f_{2} \in$ $C\left(0, T ; H^{\beta+r}(S)\right)$. Then, for all $t \leq T$,

$$
\|\tilde{\xi}(t)\|^{2} \leq b^{*}\left(\tau^{2}+M^{-2 r}\right),
$$

where $b^{*}$ is a positive constant depending only on the norms of $\xi$ and $f_{2}$ in the spaces mentioned above.

\section{BIBLIOGRAPHY}

1. J. H. Bramble and J. E. Pasciak, A boundary parametric approximation to the linearized scalar potential magnetostatic field problem, Appl. Numer. Math. 1 (1985), 493-514.

2. C. Canuto, M. Y. Hussaini, A. Quarteroni, and T. A. Zang, Spectral methods in fluid dynamics, Springer-Verlag, Berlin, 1988.

3. R. Courant and D. Hilbert, Methods of mathematical physics, Vol. 1, Interscience, New York, 1953.

4. D. Gottlieb and S. A. Orszag, Numerical analysis of spectral methods, CBMS-NSF, Regional Conference Series in Applied Mathematics, vol. 26, SIAM, Philadelphia, PA, 1977.

5. Ben-yu Guo, Spectral method for Navier-Stokes equation, Scientia Sinica 28A (1985), 11391153.

6. __ Difference method for partial differential equations, Science Press, Beijing, 1988.

7. Ben-yu Guo and He-ping Ma, Strict error estimation for a spectral method of compressible fluid flow, Calcolo 24 (1987), 263-282.

8. Ben-yu Guo, He-ping Ma, Wei-ming Cao, and Hui Huang, The Fourier-Chebyshev spectral method for solving two-dimensional unsteady vorticity equation, J. Comput. Phys. 101 (1992), 207-217.

9. G. J. Haltiner, Numerical prediction, Wiley, New York, 1971.

10. G. J. Haltiner and R. T. Williams, Numerical prediction and dynamical meteorology, Wiley, New York, 1980.

11. M. Jarraud and A. P. M. Baede, The use of spectral techniques in numerical weather prediction, Large-Scale Computation in Fluid Mechanics, Lectures in Appl. Math., vol. 22, Amer. Math. Soc., Providence, RI, 1985, pp. 1-41.

12. H. O. Kreiss and J. Oliger, Stability of the Fourier method, SIAM J. Numer. Anal. 16 (1979), 421-433.

13. Pen-yu Kuo, The convergence of spectral scheme for solving two-dimensional vorticity equation, J. Comput. Math. 1 (1983), 353-362. 
14. J. L. Lions, Quelques méthodes de résolution des problèmes aux limites non linéaires, Dunod, Paris, 1969.

15. J. L. Lions and E. Magenes, Nonhomogeneous boundary value problems and applications, Vol. 1, Springer-Verlag, Berlin, 1972.

16. Y. Maday and A. Quarteroni, Spectral and pseudospectral approximation of the Navier-Stokes equation, SIAM J. Numer. Anal. 19 (1982), 761-780.

17. J. E. Pasciak, Spectral methods for a nonlinear initial value problem involving pseudodifferential operators, SIAM J. Numer. Anal. 19 (1982), 142-154.

18. R. D. Richtmyer and K. W. Morton, Difference methods for initial-value problems, 2nd ed., Interscience, New York, 1967.

19. Qingcun Zen, Physical-mathematical basis of numerical prediction, Vol. 1, Science Press, Beijing, 1979.

Shanghai University of Science and Technology, Shanghai 201800, People's Republic OF CHINA 